\title{
Vibrations and Relaxations in a Soft Sphere Glass: Boson Peak and Structure Factors
}

\author{
H. R. Schober \\ Institut für Festkörperforschung, Forschungszentrum Jülich, D-52425 Jülich, Germany
}

(Dated: October 25, 2018)

\begin{abstract}
The dynamics of a soft sphere model glass, studied by molecular dynamics, is investigated. The vibrational density of states divided by $\omega^{2}$ shows a pronounced boson peak. Its shape is in agreement with the universal form derived for soft oscillators interacting with sound waves. The excess vibrations forming the boson peak have mainly transverse character. From the dynamic structure factor in the Brillouin regime pseudo dispersion curves are calculated. Whereas the longitudinal phonons are well defined up to the pseudo zone boundary the transverse ones rapidly get over-damped and go through the Ioffe-Regel limit near the boson peak frequency. In the high $q$ regime constant- $\omega$ scans of the dynamic structure factor for frequencies around the boson peak are clearly distinct from those for zone boundary frequencies. Above the Brillouin regime, the scans for the low frequency modes follow closely the static structure factor. This still holds after a deconvolution of the exact harmonic eigenmodes into local and extended modes. Also the structure factor for local relaxations at finite temperatures resembles the static one. This semblance between the structure factors mirrors the collective motion of chain like structures in both low frequency vibrations and atomic hopping processes, observed in the earlier investigations.
\end{abstract}

PACS numbers: PACS number(s): 61.43.Fs, 63.50.+x, 66.30.Fq 


\section{INTRODUCTION}

Despite large efforts, the vibrational dynamics of amorphous materials and glasses is still not fully understood and subject to a controversial debate. At a first sight there are many similarities between glasses and amorphous materials and their ordered counterparts, the crystals. The vibrational densities of states are normally similar and typical average frequencies or Debye temperatures do not change dramatically from one state to another. This reflects the the similarity of the short range order of both states, primarily the average nearest neighbour distance is similar. At higher, but still low, frequencies the vibrational density of states (DOS), $Z(\omega)$, in glasses exceeds the Debye value and a maximum is seen in the inelastic scattering intensity, the so called boson peak (BP). Due to disorder the sound waves are increasingly damped with increasing frequency and eventually the damping exceeds the Ioffe-Regel limit: The mean free path of the phonons diminishes below their wavelengths, the phonons are over-damped. The vibrations can still be understood by harmonic theory but their eigenvectors will have a complicated structure reflecting the structural disorder. The frequency, $\omega_{\mathrm{IR}}$, where the Ioffe-Regel limit is reached, is normally near the BP frequency, $\omega_{\mathrm{BP}}$. As in defect crystals, one finds in addition to the "band" modes localized vibrational modes above the maximum frequency of the band modes or in gaps of the spectrum. Additionally, particularly in molecular glasses, there can be the equivalent of the optical vibrational modes of crystals, albeit often broken up by disorder.

The dispute centers mainly on two issues: first,the nature of the excess of low frequency modes above the Debye value, i.e. the origin of the BP, and secondly on the properties of the phonons at higher frequencies. The term phonon is used here for an extended vibrational mode which can be classified by a $q$-value. The latter question has gained great interest by the advance of modern synchrotron sources which allow to measure structure factors in Brillouin scattering.

The frequency domain, typically around $\nu=1 \mathrm{THz}$, of the BP is accessible to a variety of spectroscopic methods like Raman [1] and neutron scattering [2]. From the temperature dependence of the measured intensities it has been deduced that the excitations are essen-

tially harmonic vibrations. The boson peak is, therefore, a maximum in $Z(\omega) / \omega^{2}$. Mostly it is not a maximum of the vibrational spectral density, $Z(\omega)$, itself.

Over the years a large number of explanations of the excess intensity and the BP have 
been proposed. First, there are some materials where some molecular unit has an inherent low frequency mode, such as in plastic crystals. These modes will trivially show as "excess" intensity in $Z(\omega)$. We will not consider these. Secondly, there are materials where the BP is a real effect of disorder effect. The model, studied here, belongs to this class it has further no molecular vibrations or low lying optic modes in its crystalline form. Some authors attribute the BP to vibrations of clusters of atoms of typical sizes [3, 4]. The origin of these clusters is unclear and they have not been identified in numerical simulations. Another popular qualitative explanation of the Boson peak is a softening of acoustic phonons by static disorder [5, 6] due to elastic Rayleigh scattering. However, estimates show that the Rayleigh contribution is too small to explain the experimental data on thermal conductivity in glasses [6]. Sometimes the BP is related to low lying optic modes of parental crystals [7, 8, 9]. Whereas the BP is a general feature of glasses such crystal structures with soft optic modes cannot be identified always. Such a mechanism is possible in some cases. A distinction optic modes, which disorder has broken into segments, and resonant vibrations is not very clear in all cases.

Recent work on harmonic lattice models demonstrated that softening of disordered force constants can smear and push to low frequencies peaks which exist in the crystalline DOS 10 , 11, 12]. In another approach the vibrations of a random distribution of atoms, interacting with a Gaussian-shaped pair potential, was studied [13] in a harmonic scalar approximation.

A different explanation is offered by the soft potential model (SPM) 14, 15]. This model gives a unified description of the glassy low energy dynamics: tunneling, local relaxation and excess in low frequency vibrations. For the latter it postulates low frequency oscillators which interact bilinearly with the sound waves and hybridize with them. Thus quasi-local vibrations, also called resonant vibrations, are created [16]. Fitting the model to the experimental data, one finds effective masses of 20-100 atomic masses for the entities moving in these effective soft potentials [17]. The sound waves in turn will be damped by resonant scattering with these modes [18]. For low frequencies the SPM predicts an increase of the excess of the DOS as $Z_{\text {excess }}(\omega) \propto \omega^{4}$. This rapid increase of $Z_{\text {excess }}(\omega)$ will lead to level repulsion and an eventual cross over to $Z_{\text {excess }}(\omega) \propto \omega$ [19]. Recently it has been shown that the interaction between the soft oscillators in harmonic approximation causes unstable vibrations, negative eigenvalues. The structural configuration becomes unstable. The response of the glass is then a local relaxation to a new stable configuration which always exist 
due to anharmonicity. The harmonic spectrum in this new stable configuration exhibits a $\mathrm{BP}$ with a universal shape as it is observed in experiment. In particular the shape of the $\mathrm{BP}$ is independent of the actual magnitude of the anharmonicity [20].

Computer simulations of a soft sphere glass (SSG) [21, 22] confirmed the existence of low frequency quasi-localized vibrations with effective masses ranging from ten atomic masses upwards. The modes were centered at structural irregularities with large local strains [23]. Regions of local strain have also been observed in earlier computer simulations [24]. Similar effective masses have since been observed in many different simulations, e.g. $\mathrm{SiO}_{2}$ [25], Se [26] in Ni-Zr [27], Pd-Si [28], Ni-B [29], amorphous ice [30] and amorphous and quasi-crystalline $\mathrm{Al}-\mathrm{Zn}-\mathrm{Mg}$ [31]. In an earlier simulation of amorphous silicon low frequency localized vibrations have been observed at coordination defects [32]. It should be noted that computer simulations with the usual periodic boundary conditions have a lower cutoff, $\omega_{\text {min-sound }}$ for the sound waves, which is given by the maximal wavelength fitting into the periodicity volume. Quasi localized vibrations with frequencies $\omega<\omega_{\min -\text { sound }}$ appear, therefore, in simulations as truly localized vibrations which can be used as an easy way to identify them. For $\omega>\omega_{\text {min-sound }}$ hybridisation with the sound waves occurs and the local vibrations are delocalized, they become quasi-localized. This will lead to a small shift in $Z_{\text {excess }}(\omega)$ but does not affect the number of excess modes. For a more detailed discussion see [33, 34]. For frequencies $\omega>\omega_{\text {min-sound }}$ it is still possible to change from the basis of exact harmonic eigenvectors to a basis of sound waves and local vibrations with a small bilinear interaction 34]. The latter basis is the one used in the SPM.

High resolution synchrotron scattering made it possible to observe the dynamic structure factor, $S(q, \omega)$, in Brillouin scattering [35]. For a recent review see [36]. As in crystals, At the lowest frequencies glasses have well defined longitudinal and transverse sound waves. Only the longitudinal one is observed in Brillouin scattering at low $q$. At the lowest frequencies the inelastic scattering function or the dynamic structure factor shows, as function of frequency for a given $q$, only one peak which is ascribed to the longitudinal sound wave. At higher frequencies a second peak, ascribed to the transverse sound is observed [37]. This can be understood considering that in a disordered system a transverse sound wave acquires a longitudinal component and vice versa. Fitting a damped harmonic oscillator to $S(q, \omega)$ in amorphous silica [38] and glycerol [39], one group concluded in that propagating collective excitations exist in glasses at high $q$-values, i. e. frequencies far above the BP. From 
measurements of densified silica another group [40, 41] reach the opposite conclusion that the BP marks the end of the propagating acoustic modes.

This question can at present not be answered unambiguously from experiment. Additional insight can be gained by computer simulation. Molecular dynamics simulations of amorphous silica gave structure factors in good agreement with experiment. A fit with a damped harmonic oscillator function gave a damping of the longitudinal mode $\gamma(q) \propto q^{2}$ for frequencies above the BP [42, 43].

The aim of the present paper is to use a simple model glass, representative for dense packed metallic glasses, to see what information can be gained from the dynamic structure both in the Brillouin regime and for higher $q$-values. For this we extend our previous study of the soft sphere glass [21, 22, 34]. This glass has the advantage that there is no parent crystal with optical modes and no torsional motion as one has in silica which is built from more or less rigid $\mathrm{SiO}_{4}$-tetrahedra. In the earlier studies [34] we found in a normal mode analysis that the Ioffe-Regel limit is reached for the transverse acoustic modes near the BP. For the lowest frequency in the calculation the width of the phonon line could be split into two approximately equal parts attributed to static disorder and to resonance scattering with QLV.

\section{COMPUTATIONAL DETAILS}

The soft sphere glass (SSG) is described by an inverse sixth-power potential

$$
u(r)=\epsilon\left(\frac{\sigma}{r}\right)^{6}+A\left(\frac{r}{\sigma}\right)^{4}+B
$$

To simplify the computer simulation and normal-mode analysis, the potential is cut off at $r / \sigma=3.0$, and then shifted by a polynomial, $A(r / \sigma)^{4}+B$, where $A=2.54 \cdot 10^{-5} \epsilon$ and $B=-3.43 \cdot 10^{-3} \epsilon$ were chosen so that the potential and the force are zero at the cutoff. This form of the shifting function was chosen so that its effect is negligible near $r / \sigma=1.0$. Quantities such as the pressure and average potential energy will be changed by a few percent as a result of this truncation, but any changes in the equilibrium structure will be small. Without loss of generality one can set $\epsilon=\sigma=m=1$. Where we do not explicitly state the units we infer these "system units". Note that this soft sphere potential is much softer than the often used one with $u_{12} \propto(\sigma / r)^{1} 2$. 
The calculations were done using the samples prepared earlier for investigations of vibrations [34] and relaxations [44]. These had been prepared by the following procedure. Liquid configurations of 5488 soft spheres were produced via constant-energy molecular dynamics (MD) simulation with cubic periodic boundary conditions at a density, $\rho \sigma^{3}=1.0$, and temperature, $k T / \epsilon \approx 0.54$ (about 2.5 times the melting temperature at this density 45]). For the simulation, we used the velocity-Verlet algorithm with a time step of 0.04 - in units of $\left(m \sigma^{2} / \epsilon\right)^{1 / 2}$. The liquid was first quenched within the MD simulation by velocity rescaling to a reduced temperature of about $0.005 T_{g}$. The quench rate was about $0.015 k /\left(m \sigma^{2} \epsilon\right)^{1 / 2}$. After the MD quench, each sample was heated to $0.05 T_{g}$ and aged for several 1000 further MD time steps to stabilize the potential energy and to avoid spurious minima. Each system was finally quenched to zero temperature using a combination the steepest-descent/conjugategradient algorithm of our version of the Harwell code DEVIL, the metals version of the HADES code. To improve statistics 11 different configurations of 5488 atoms, created in this way, were analyzed.

\section{VIBRATIONS}

The vibrational dynamics was calculated in classical harmonic approximation from the force constant matrix of the $T=0$ minimum configuration. The numerically exact minimization of the potential energy prevents the occurrence of spurious unstable modes. The elements of the force constant matrix are given by

$$
D_{\alpha \beta}^{m n}=\frac{\partial^{2} u\left(\left|\mathbf{R}^{m}-\mathbf{R}^{n}\right|\right)}{\partial R_{\alpha}^{m} \partial R_{\beta}^{n}}, \quad m \neq n .
$$

Different from our previous work [34] all eigenvalues, $\left(\omega^{\sigma}\right)^{2}$, and eigenvectors, $\mathbf{e}^{n}(\sigma)$, were calculated by direct diagonalisation.

To localize the BP we calculate the vibrational density of states from the frequencies of the $3 N-3$ vibrational modes $\sigma$ as

$$
Z(\omega)=\left\langle\frac{1}{3 N-3} \sum_{\sigma} \delta\left(\omega-\omega^{\sigma}\right)\right\rangle
$$

where $\delta$ is the discretized $\delta$ function and \langle\rangle stands for the averaging over configurations. Within the numerical accuracy the resulting spectrum is unchanged from Fig. 1 of [34]. The maximal frequency is $\omega_{\max } \approx 12.6$. There is a slight dip around $\omega=9.5$ which is characteristic for the chosen soft sphere glass. It reflects the factor three between the longitudinal 


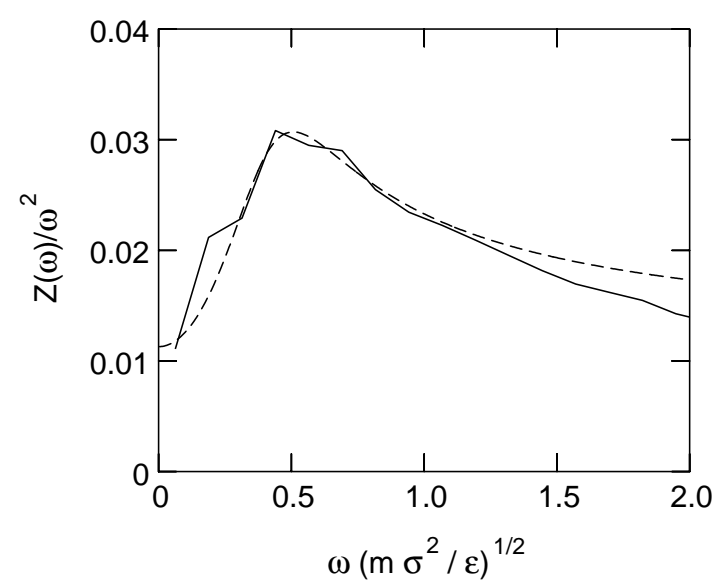

FIG. 1: Vibrational spectrum divided by $\omega^{2}$ versus $\omega$. The solid line gives the simulation results, corrected by a Debye contribution at the lowest frequencies. The dashed line is a fit with the model of Ref. [20].

and transverse sound velocities. It disappears for harder models, i. e. a larger exponent of the $\sigma / r$-term in Eq. 1 .

Fig. [1 (solid line) shows the calculated $Z(\omega) / \omega^{2}$, corresponding to the BP. From the size of our simulation cell we calculate from the elastic constants $\omega_{\text {min-sound }}=0.52$ and 1.76 for the longitudinal and transverse sound, respectively. The simulated density of states has no sound waves below these cutoffs. To compensate for this we added for the lower frequencies a Debye contribution to $Z(\omega)$, calculated from the elastic constants. The dashed line shows the theoretical estimate for the model of interacting soft oscillators and sound waves [20]

$$
Z_{\text {theor }}(\omega)=Z_{\mathrm{D}}(\omega)+Z_{\text {excess }}(\omega)
$$

where $Z_{\mathrm{D}}(\omega)$ is the Debye spectrum and $Z_{\text {excess }}(\omega)$ the excess causing the BP. There is very good agreement between the two curves up to $\omega=2 \omega_{\mathrm{BP}}$. The deviation at higher frequencies has to be expected. We arrive, in agreement with the earlier work, at an estimate $\omega_{\mathrm{BP}}=0.5$ which is near to $\omega_{\min -\text { sound }}$ of the transverse branch and well below the corresponding value of the longitudinal branch. The considered soft sphere glass, therefore, represents an interesting example of a material where one has a low lying pronounced BP and a strong separation of the two branches of sound waves. This distinguishes it from the Lennard-Jones glass.

In classical harmonic approximation the dynamic structure factor can be written as

$$
S(q, \omega)=\frac{1}{N \omega}\left\langle\sum_{\sigma}\left|\sum_{n}\left(\mathbf{q} \cdot \mathbf{e}^{n}(\sigma)\right) \exp \left(i \mathbf{q} \cdot \mathbf{R}^{n}\right)\right|^{2} \delta\left(\omega-\omega^{\sigma}\right)\right\rangle .
$$


where $\delta$ is the discretized $\delta$ function and \langle\rangle stands for the averaging over configurations and directions of q. Here we omitted the temperature dependent factor $k T / \hbar \omega$, the high temperature limit of the phonon occupation number. The inelastic scattering intensity is given by

$$
I(q, \omega) \propto \frac{k T}{\omega} S(q, \omega) .
$$

Often the structure factor is defined including the temperature factor, it is then directly proportional to the inelastic scattering intensity. In our previous paper [34] we used a definition excluding the factor $1 / \omega$ in Eq. 5 .

$$
S_{\mathrm{FT}}(q, \omega)=\omega S(q, \omega)
$$

This corresponds to a projection of the Fourier transform of the eigenvectors. For broad distributions in $\omega$ the different definitions will lead to shifts in the maximum and with.

In analogy with the usual structure factor which projects out the longitudinal components of the vibrations one can define a transverse one as

$$
S_{\mathrm{tr}}(q, \omega)=\frac{1}{N \omega}\left\langle\sum_{\sigma}\left|\sum_{n}\left(\mathbf{q} \times \mathbf{e}^{n}(\sigma)\right) \exp \left(i \mathbf{q} \cdot \mathbf{R}^{n}\right)\right|^{2} \delta\left(\omega-\omega^{\sigma}\right)\right\rangle .
$$

It gives, for small $q$, access to the transverse vibrations which are not observable in the scattering experiments. We study the structure factors both in the Brillouin regime, i.e. below the first peak of the static structure factor (in our system at $q_{\mathrm{FP}}=7 \sigma^{-1}$ see Fig. (5)), and above for large $q$ values.

\section{A. Brillouin regime}

As done by other authors we do constant- $q$ scans of the dynamic structure factor and define the maxima in $\omega$ as phonon frequencies and derive this way a pseudo dispersion curve. This is shown in Fig. 2 for both the longitudinal and transverse branches determined from Eqs. 5 and 8, respectively. The error bars indicate the full width half maximum.

One sees a very different behaviour of the two branches. Whereas the longitudinal one is well defined well beyond the pseudo zone boundary $q_{\mathrm{ZB}}=0.5 \cdot q_{\mathrm{FP}}$, the transverse one becomes rapidly over-damped. The different broadenings of the two branches indicate a large difference in their coupling to the BP-modes. This is seen even more clearly when we define the phonons from the scattering intensity, i.e. include the factor $k T / \omega$. The 


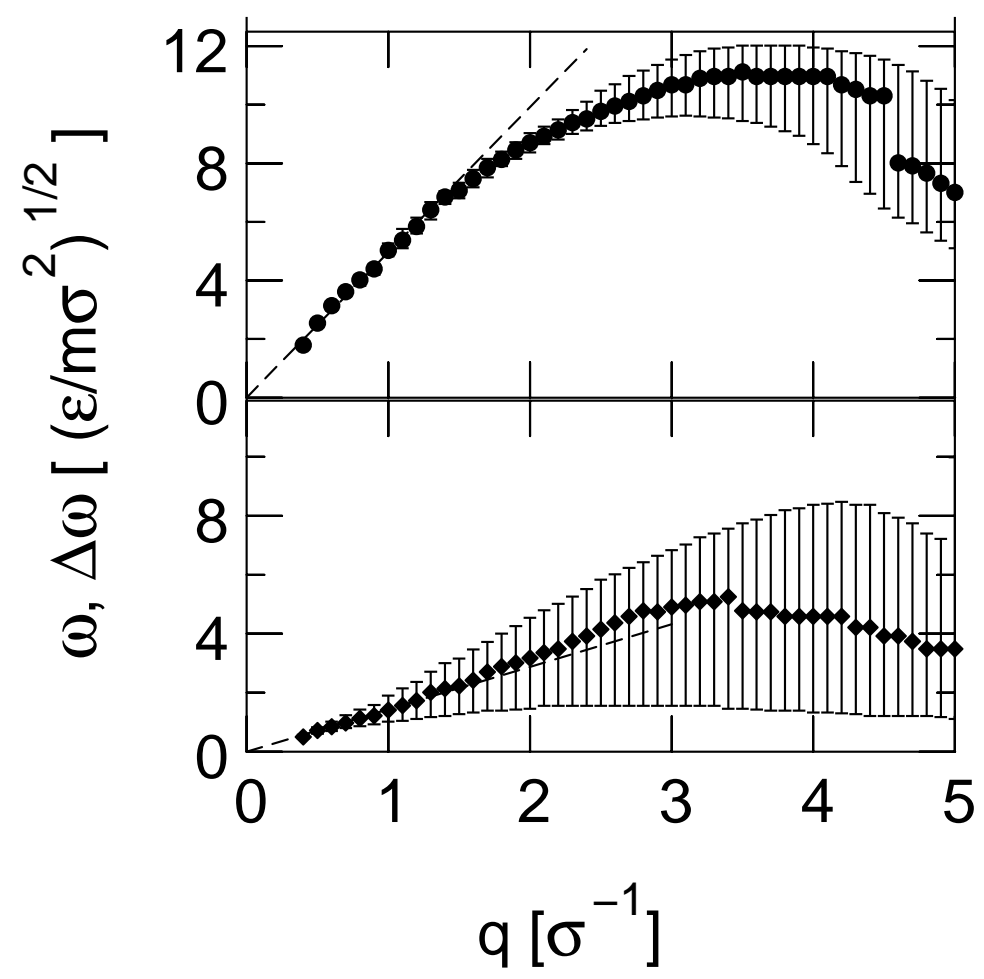

FIG. 2: Pseudo dispersion curves in the soft sphere glass (longitudinal:top transverse:bottom) calculated from the structure factor without the phonon occupation factor. The error bars indicate the full width at half height. The dashed line gives the linear dispersion given by the sound velocities, calculated from the elastic constants of the glass.

longitudinal branch is only slightly affected whereas the transverse one broadens even more and above $q=1.8 \sigma^{-1}$ the maximal intensity is no longer at some ill defined phonon line but drops to the BP frequency. This indicates a transverse nature of the BP. It should be noted that the frequencies of both branches at the pseudo zone boundary are much higher than the BP-frequency $\omega_{\mathrm{BP}}$. This certainly does not justify an explanation of the BP in terms of soft zone edge phonons. For low $q$, both branches show a linear dispersion according to $\omega=c q$. The transverse branch shows above $q=1,5 \sigma^{-1}$ an apparent slightly positive dispersion as has been seen for the longitudinal branch in $\mathrm{SiO}_{2}$ [42].

Fig. 3 gives the broadening of the pseudo dispersion for different weightings with $1 / \omega$, corresponding to the definitions via the scattering intensity (Eq. [6), the correlation function (Eq. 5. or a Fourier transform of the eigenstates (Eq. 7). As to be expected the definition does not change the broadening at the low $q$-values but is essential above the Ioffe-Regel 


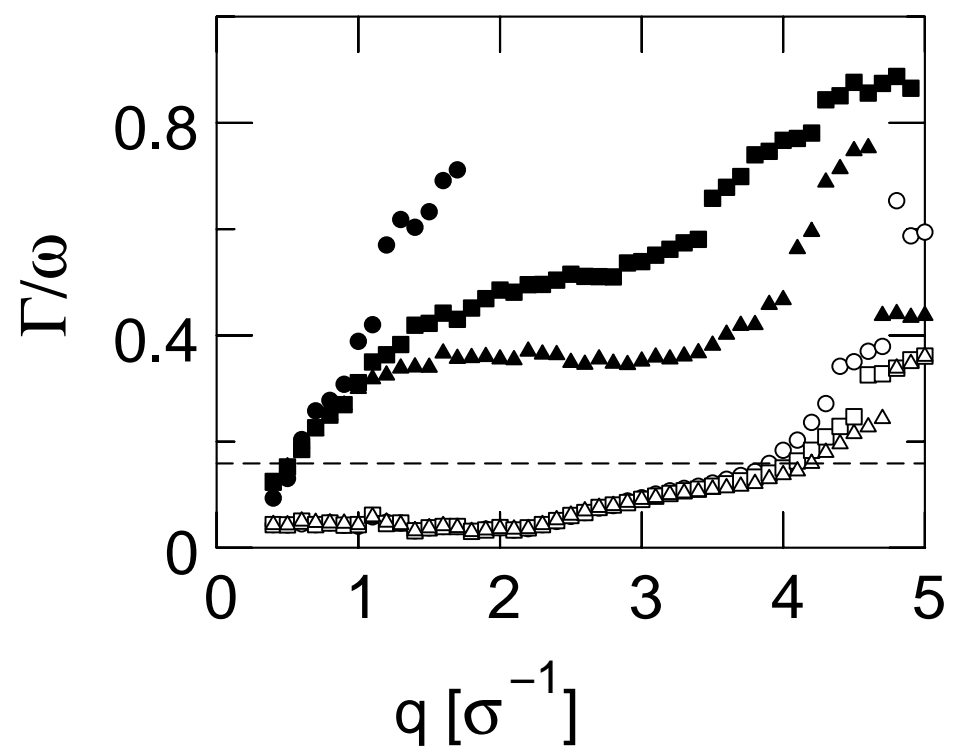

FIG. 3: Halfwidth-halfmaximum divided by $\omega$ versus omega, calculated from the dynamic structure factor with different $\omega$-weighting. Open and full symbols longitudinal and transverse branches, respectively; squares: weighting according Eq. [5] circles: Eq. [6] triangles: Eq. 7 The dashed line indicates the Ioffe-Regel limit $\Gamma(q)=\omega(q) / 2 \pi$.

limit when $\Gamma(\omega)>\omega / 2 \pi$. The longitudinal branch goes through $\omega_{\mathrm{IR}}$ at $q=4 \sigma^{-1}$ beyond which value it rises rapidly as $q \rightarrow q_{\mathrm{FP}}$. The transverse branch goes through $\omega_{\mathrm{IR}}$ already at $q=0.5 \sigma^{-1}$. There are three apparent regions in $\Gamma(q)$, best seen in the values derived $S_{\mathrm{FT}}(q, \omega)$. At the lowest $q$ the width, $\Gamma(q)$, increases with a power of $q^{2}$ or larger, followed by $\Gamma(q) \propto q \propto \omega(q)$ and finally there is an upturn for $q \rightarrow q_{\mathrm{FP}}$.

The soft potential model predicts $\Gamma(q) \propto q^{4}$ for $\omega(q)<\omega_{\mathrm{BP}}$ which is derived from the initial increase of the excess vibrational $\operatorname{DOS} Z_{\text {excess }}(\omega) \propto \omega^{4}$ [18]. Unfortunately, the low value of $\omega_{\mathrm{BP}}$ in the investigated soft sphere glass excludes this regime from our simulation. The simulation cell would have to be large enough not only to allow small $q$-values it would also have to accommodate several mean free paths of these phonons. Otherwise the phonons would be scattered by a periodic repetition of equal defects instead of a distribution of different defects. For $q<1 \sigma^{-1}$ we observe for the transverse branch an increase $\Gamma(q) \propto q^{2}$ as 
has been observed earlier for strongly frustrated systems [46]. The section where $\Gamma(q) \propto \omega q$ is broadly compatible with the increase of the total $Z(\omega) \propto \omega$ for $\omega>\omega_{\mathrm{BP}}$. Above the BP the transverse modes are strongly mixed with the quasi-localized vibrations and amongst each other. Therefore all transverse modes can be considered as scattering centers.

Our results for the pseudo dispersion curves agree with the earlier calculation by Caprion

et al. [47]. There is, however, a marked difference in the calculated widths. This might be due both to the better resolution in the present work and also to the different procedure used to calculate $\Gamma(\omega)$. We define the width directly from the width at half maximum whereas Caprion uses a Lorentzian fit. It was noted earlier that the peaks in $S(q, \omega)$ are asymmetric 34]. This makes a fit dependent on details of the adopted fitting procedure.

\section{B. Structure factor at high $q$-values}

The dynamic structure factor in the Brillouin regime provides only rather limited information on the structure of the vibrational modes, even if one includes the experimentally hardly measurable transverse branch. Additional information can be gained if one includes $q$-values beyond the first diffraction peak where $S(q, \omega)$ can be measured by inelastic neutron scattering. As seen from Fig. 4 the dynamic structure factor exhibits even for this simple material a rich structure. For the sake of clarity we plotted $S_{\mathrm{FT}}(q, \omega) / q^{2} Z(\omega)$. Taking constant $\omega$ cuts of $S(q, \omega)$ one gets distinct patterns for the average of the modes with that frequency.

In Fig. 5 a we show four such constant omega scans: for $\omega=\omega_{\mathrm{BP}}$, for the top of the apparent acoustic phonon in Fig. 2, for $\omega$ near the Einstein average and for localized vibrations. For comparison the static structure factor, $S_{\text {stat }}(q)$, is also shown. Comparing the low frequency and high frequency vibrations one observes a clear shift of phase with respect to the static structure factor. The low frequency vibrations are in phase but the localized ones are in anti-phase to $S_{\text {stat }}(q)$. In simple densely packed materials, e.g. metals, the nearest neighbour coupling dominates. Localized vibrations are vibrations of neighbouring atoms against each other and this is seen as phase shift against $S_{\text {stat }}(q)$. Low frequency vibrations on the other hand must not involve significant changes of the nearest neighbour distance and therefore show no phase shift. The intermediate frequency modes $(\omega=3.8$ and 8$)$ show a mixed behaviour. The lower of the two frequencies corresponds to the high frequency 


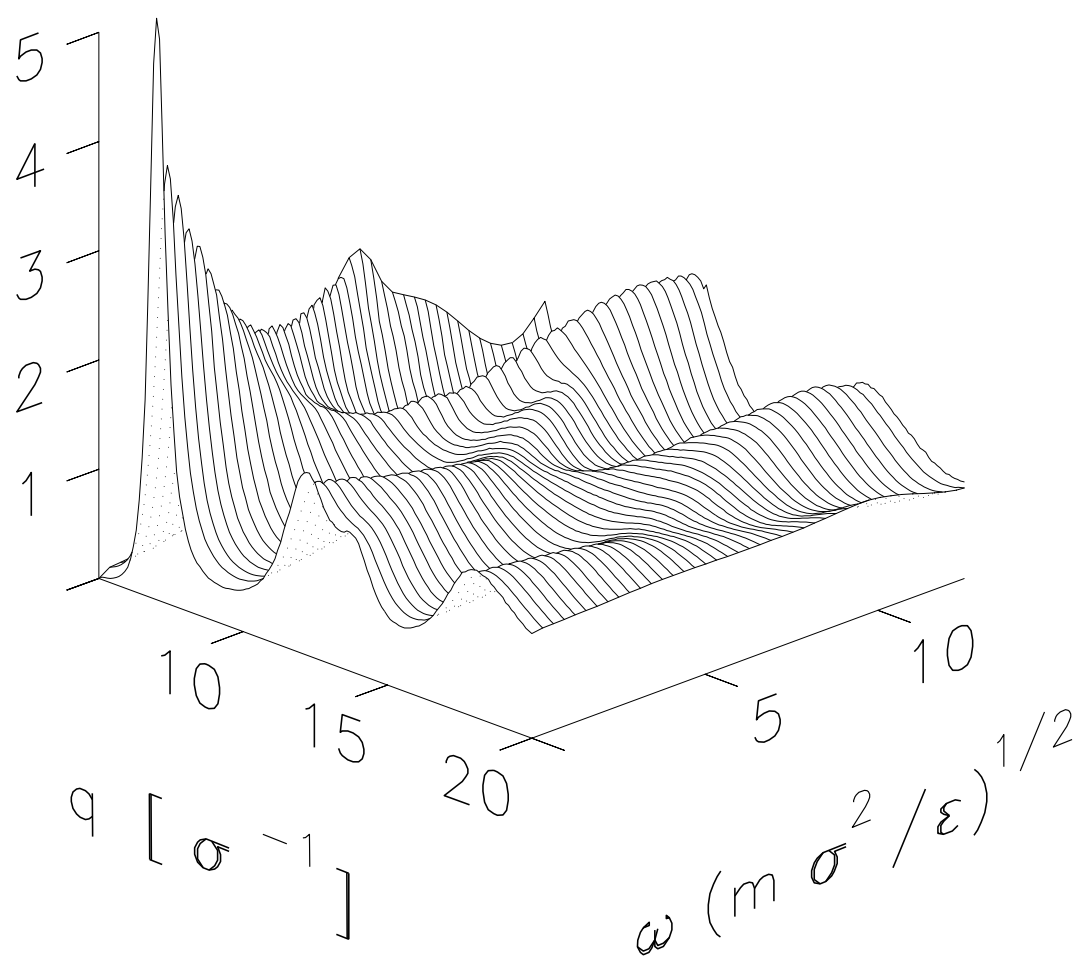

FIG. 4: Dynamic structure factor $S_{F T}(q, \omega) / q^{2} Z(\omega)$. For $\omega=0$ the static structure factor was taken.

edge of the transverse mode. In the constant-omega scan one sees a narrow line at low $q$ from the longitudinal phonon, a flat maximum around $q_{\mathrm{FP}}$ and a weak modulation, clearly distinct from $S_{\text {stat }}(q)$ at larger $q$. For the other scan with $\omega=8$, which is above the apparent maximal transverse phonon frequencies, one sees again the narrow peak of the longitudinal phonon, now at a higher $q$-value, and additionally a broader peak marking the descent of the pseudo-dispersion for $q \rightarrow q \mathrm{FP}$. The oscillations for larger $q$ are out of phase with $S_{\text {stat }}(q)$ and damped compared to the curve for the localized vibrations. Clearly the character of the modes with $\omega \approx \omega_{\mathrm{BP}}$ is distinct from the one of the zone boundary modes. Using our previous results, we take a closer look at the modes responsible for the BP.

In Ref. 34] we deconvoluted the exact low frequency eigenvectors of the present system into extended sound wave like modes and local modes with a weak bilinear interaction. This was done by rotating the basis of modes in a narrow energy range. This way we extracted optimally localized vibrational modes and automatically gained the proper number of extended phonons. The total number of modes is preserved and the number of modes in a given frequency interval is only marginally altered. The resulting extended and localized 


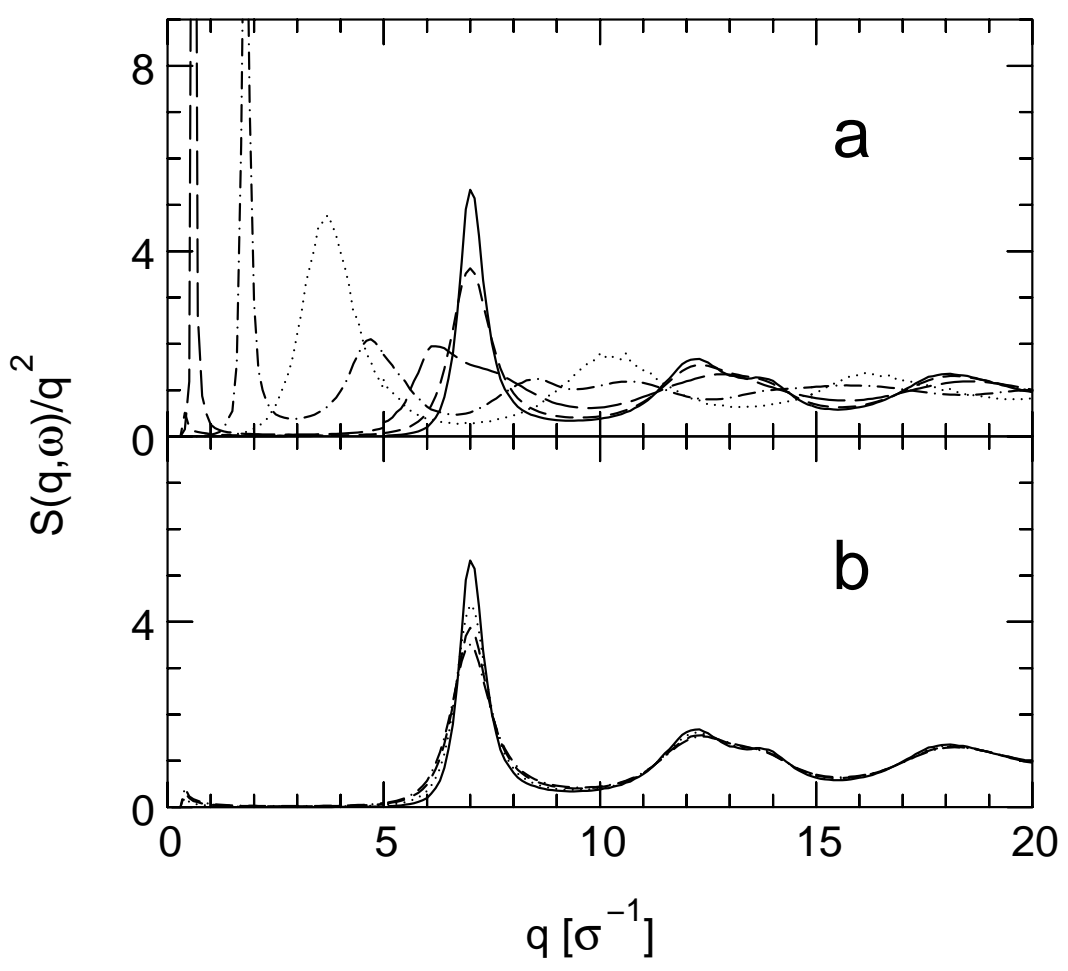

FIG. 5: Constant frequency scans of the dynamic structure factor. Solid line: static structure factor, a: dashed line $\omega=0.6 \approx \omega_{\mathrm{BP}}$, long dashed line $\omega=3.8$, dotted line $\omega=8$, dash dotted line $\omega=12 ; \mathrm{b}: \omega=0.6 \approx \omega_{\mathrm{BP}}$, dashed line averaged as in a, dotted line: extended mode, dash dotted line: local mode.

modes, are no longer eigenvectors of the dynamic matrix but have a weak bilinear interaction. This is the picture used in the soft potential model. In Fig. 5 b we compare $S_{\text {stat }}(q)$ with constant- $\omega$ scans at $\omega \approx \omega_{\mathrm{BP}}$ for the the true harmonic eigenmodes and the deconvoluted extended and local parts. It is not surprising that the curves of the deconvoluted extended modes follows $S_{\text {stat }}(q)$ even more closely than the one of the eigenmodes. This property of sound was was suggested by Buchenau et al. [48] as a distinguishing feature between them and local vibrations. This difference is clearly not very pronounced in the present case. For the deconvoluted local low frequency modes the first peak is certainly reduced but still quite pronounced. For larger $q$ the scans of the extended and local modes are hardly distinguishable. This somewhat surprising result is a consequence of the strongly collective nature of the local vibrations, which form the cores of the quasi-localized vibrations seen in $Z_{\text {excess. }}$. These modes are collective motions of chains of 10 and more atoms [21, 34, 49]. 
Such modes are typical fore densely packed metallic glasses.

\section{RELAXATIONS}

Besides vibration one observes in a glass aperiodic jumps of groups of atoms, local relaxations. Such jumps give e. g. rise to the telegraph noise in point contacts and are thought to be the elementary process in diffusion [50]. In our previous study of relaxations in the soft sphere glass we found them to be closely correlated to the quasi-localized vibrations. They are again collective motions of chains of atoms [44, 49]. At low temperatures any given atom moves in such a jump only a fraction of the nearest neighbour distance. One can observe these collective jumps in a molecular dynamics simulation by monitoring the total displacement of all atoms as function of time

$$
\Delta R(t)=\sqrt{\sum_{n}\left(\mathbf{R}^{n}(t)-\mathbf{R}^{n}(0)\right)^{2}}
$$

where $\mathbf{R}^{n}(t)$ is the position vector of particle, $n$, at time, $t$, and $\mathbf{R}^{n}(0)$ is the one at the starting or reference configuration. $\Delta R(t)$ oscillates due to the vibrations and changes due to relaxations, i.e. due to the transitions from one local energy minimum to another. At low

temperatures $T \approx 0.05 T_{\mathrm{g}}$, the maximal distance an individual atom travels in these jumps is only $0.3 \sigma$, about a quarter of $R_{N N}$. Increasing the temperature by a factor of four the jumps seen at the lower temperature can no longer be resolved and new jumps over larger distances are observed.

All observed relaxations were collective jumps localized to 10 and more atoms forming twisted chain-like structures with some side branching [44]. This collectivity is again reflected in the relaxational structure factor, defined in analogy with the vibrational (Eq. 7) one

$$
S_{\text {rel }}(Q)=\frac{1}{Q^{2}}\left\langle\left|\sum_{n} \mathbf{Q} \Delta \mathbf{R}^{n} \exp \left(i \mathbf{Q} \mathbf{R}^{n}\right)\right|^{2}\right\rangle
$$

where $\Delta \mathbf{R}^{n}$ is the jump vector of atom $n$ and $\langle\ldots\rangle$ denotes averaging over angle and relaxations. The relaxational structure factor is similar to the corresponding curve for the low frequency vibrations. It follows its static counterpart closely, independent of the jump lengths. The smoothing out of the higher peaks is more pronounced for the longer jumps occurring predominantly at higher temperatures. Comparing Figs. 5 a and 6 one sees the close correlation between quasi-localized vibrations and atomic jumps expressed which we established earlier by correlating vibrational eigenvectors an jump vectors [44]. 


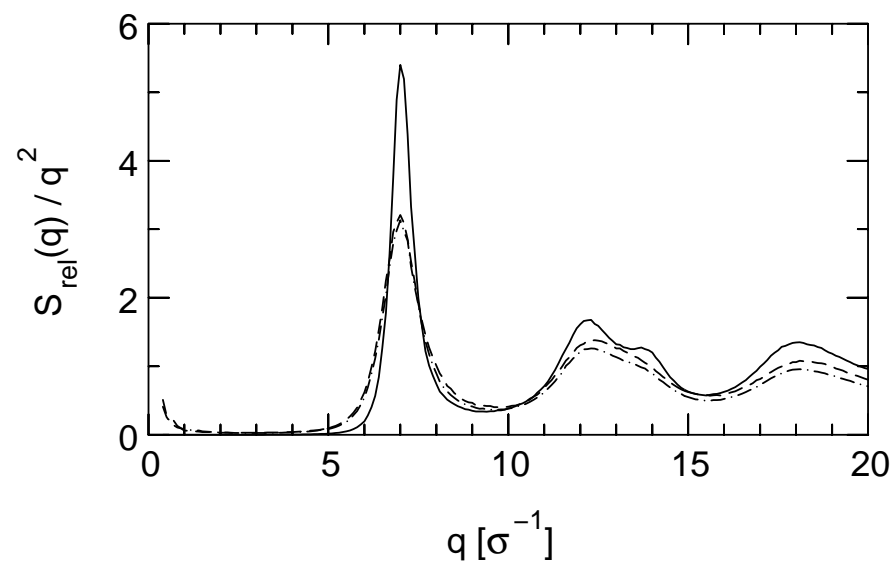

FIG. 6: Structure factor of local relaxations at temperatures up to $0.15 T_{\mathrm{g}}$. Full line: static structure factor, dashed line: average over relaxations with $\Delta R<5 \sigma$, dash dotted line: average over relaxations with $\Delta R<10 \sigma$.

\section{CONCLUSIONS}

The vibrational spectrum of the $1 / r^{6}$-soft-sphere glass shows a pronounced boson peak. The boson peak frequency is very low at about $5 \%$ of the maximal frequency. The shape of the boson peak is in accordance with the one obtained from a model of interacting soft oscillators and sound waves. Calculating pseudo dispersion curves one finds a different behaviour for longitudinal and transverse phonons. Whereas the longitudinal ones are well developed in the whole of the first pseudo Brillouin zone the transverse ones become overdamped near the boson peak frequency. Perhaps not surprisingly, but often forgotten, this shows that depending on the underlying atomic structure the two branches of sound waves vary in their behaviour as function of $q$. In the studied model glass, the boson peak vibrations have predominantly transverse character.

From the structure factor at higher $q$ we find that the boson peak vibrations are distinct from the zone boundary modes. They closely follow the structure of the static structure factor. Splitting the exact eigenmodes into extended phonons and localized modes one finds that both components again follow the large $q$ part of the static structure factor. The larger extent of the phonons is seen as a larger amplitude at the first peak. This semblance of the structure factors can be understood from the collectivity of the localized vibrations, the cores of the quasi-localized vibration. As we found earlier these modes are collective 
vibrations of chains of ten and more atoms. These chains move in such way that the nearest neighbour distances do not change markedly.

The structure factors of local relaxations again resembles the static one and is closely related to the one of the boson peak vibrations. This is seen as a consequence of of the similar structure of the low frequency localized vibrations which produce the boson peak in inelastic scattering and the collective jump processes which are the elementary step in diffusion and relaxation in metallic glasses.

[1] G. Winterling, Phys. Rev. B 12, 2432 (1975).

[2] U. Buchenau, H. M. Zhou, N. Nücker, K. S. Gilroy, and W. A. Phillips, Phys. Rev. Lett. 60, 1318 (1988).

[3] V. K. Malinovsky, V. N. Novikov, and A. P. Sokolov, Phys. Lett. A 153, 63 (1991).

[4] T. Pang, Phys. Rev. B 45, 2490 (1992).

[5] C. Masciovecchio, V. Mazzacurati, G. Monaco, G. Ruocco, T. Scopigno, F. Sette, P. Benassi, A. Cunsolo, A. Fontana, M. Krisch, et al., Phil. Mag. B 79, 2013 (1999).

[6] S. R. Elliott, Europhys. Lett. 19, 201 (1992).

[7] M. T. Dove, M. J. Harris, A. C. Hannon, J. M. Parker, I. P. Swainson, and M. Gambhir, PRL 78, 1070 (1997).

[8] V. N. Sagaev, E. N. Smelyanskaya, V. G. Plotchinichenko, V. V. Koltashev, A. A. Volkov, and P. Pernice, J. Non-Cryst. Solids 248, 141 (1999).

[9] J. A. F. Jr, V. Shanabrook, and U. Strom, J. Non-Cryst. Solids 77\&78, 1125 (1985).

[10] G. D. W. Schirmacher and C. Ganter, Phys. Rev. Lett. 81, 136 (1998).

[11] S. N. Taraskin, Y. L. Loh, G. Natarajan, and S. R. Elliott, Phys. Rev. Lett. 86, 1255 (2001).

[12] J. W. Kantelhardt, S. Russ, and A. Bunde, PRB 63, 064302 (2001).

[13] T. S. Grigera, V. Martin-Mayor, G. Parisi, and P. Verocchio, J. Phys.: Condens. Matter 14, 2167 (2002).

[14] V. G. Karpov, M. I. Klinger, and F. N. Ignatiev, Sov. Phys. JETP 57, 439 (1983).

[15] M. A. Il’in, V. G. Karpov, and D. A. Parshin, Sov. Phys. JETP 65, 165 (1983).

[16] A. A. Maradudin, E. W. Montroll, G. H. Weiss, and I. P. Ipatova, Theory of Lattice Dynamics in the Harmonic Approximation, vol. 3 of Solid State Physics Suppl. (Academic Press, New 
York, 1971).

[17] U. Buchenau, Y. M. Galperin, V. L. Gurevich, and H. R. Schober, Phys. Rev. B 43, 5039 (1991).

[18] U. Buchenau, Y. M. Galperin, V. L. Gurevich, D. A. Parshin, M. A. Ramos, and H. R. Schober, Phys. Rev. B 46, 2798 (1992).

[19] V. L. Gurevich, D. A. Parshin, J. Pelous, and H. R. Schober, Phys. Rev. B 48, 16318 (1993).

[20] V. L. Gurevich, D. A. Parshin, and H. R. Schober, Phys. Rev. B 67, 094303 (2003).

[21] B. B. Laird and H. R. Schober, Phys. Rev. Lett. 66, 636 (1991).

[22] H. R. Schober and B. B. Laird, Phys. Rev. B 44, 6746 (1991).

[23] V. A. Luchnikov, N. N. Medvedev, Y. I. Naberukhin, and H. R. Schober, Phys. Rev. B 62, 3181 (2000).

[24] S.-P. Chen, T. Egami, and V. Vitek, Phys. Rev. B 37, 2440 (1988).

[25] W. Jin, P. Vashishta, R. K. Kalia, and J. P. Rino, Phys. Rev. B 48, 9359 (1993).

[26] C. Oligschleger and H. R. Schober, Physica A 201, 391 (1993).

[27] J. Hafner and M. Krajci, J. Phys. Condens. Matter 6, 4631 (1994).

[28] P. Ballone and S. Rubini, Phys. Rev. B 51, 14962 (1995).

[29] L. D. V. Ee, B. J. Thijsse, and J. Sietsma, J. Non-Cryst. Solids 205-207, 641 (1996).

[30] M. Cho, G. R. Fleming, S. Saito, I. Ohmine, and R. M. Stratt, J. Chem. Phys. 100, 6672 (1994).

[31] J. Hafner and M. Krajci, J. Phys. Condens. Matter 5, 2489 (1993).

[32] R. Biswas, A. M. Bouchard, W. A. Kamitakahara, G. S. Grest, and C. M. Soukoulis, Phys. Rev. Lett. 60, 2280 (1988).

[33] H. R. Schober and G. Ruocco, Phil. Mag. B, in print.

[34] H. R. Schober and C. Oligschleger, Phys. Rev. B 53, 11469 (1996).

[35] P. Benassi, M. Krisch, C. Masciovecchio, G. Monaco, G. Ruocco, F. Sette, and R. Verbeni, Phys. Rev. Lett. 77, 3835 (1996).

[36] G. Ruocco and F. Sette, J. Phys.: Condens. Matter 13, 9141 (2001).

[37] T. Scopigno, E. Pontecorvo, R. D. Leonardo, M. Kirsch, G. Monaco, G. Ruocco, B. Ruzicka, and F. Sette, J. Phys.: Condens. Matter 15, S1269 (2003).

[38] O. Pilla, A. Cunsolo, A. Fontana, C. Masciovecchio, G. Monaco, M. Montagna, G. Ruocco, T. Scopigno, and F. Sette, Phys. Rev. Lett. 85, 2136 (2000). 
[39] C. Masciovecchio, A. Mermet, G. Ruocco, and F. Sette, Phys. Rev. Lett. 85, 1266 (2000).

[40] M. Foret, R. Vacher, E. Courtens, and G. Monaco, Phys. Rev. B 66, 024204 (2002).

[41] E. Courtens, M. Foret, B. Hehlen, B. Rufflé, and R. Vacher, J. Phys.: Condens. Matter 15, S1279 (2003).

[42] R. Dell'Anna, G. Ruocco, M. Sampoli, and G. Viliani, Phys. Rev. Lett. 80, 1236 (1998).

[43] S. N. Taraskin and S. R. Elliott, Phys. Rev. B 61, 12031 (2000).

[44] C. Oligschleger and H. R. Schober, Phys. Rev. B 59, 811 (1999).

[45] W. G. Hoover, D. A. Young, and R. Grover, J. Chem. Phys. 56, 2207 (1972).

[46] L. Angelani, M. Montagna, G. Ruocco, and G. Viliani, PRL 84, 4874 (2000).

[47] D. Caprion, P. Jund, and R. Jullien, Phil. Mag. B 77, 313 (1998).

[48] U. Buchenau, A. Wischnewski, D. Richter, and B. Frick, Phys. Rev. Lett. 77, 4035 (1996).

[49] H. R. Schober, C. Oligschleger, and B. B. Laird, J. Non-Cryst. Sol. 156, 965 (1993).

[50] F. Faupel, W. Frank, M.-P. Macht, H. Mehrer, V. Naundorf, K. Rätzke, S. K. Sharma, H. R. Schober, and H. Teichler, Rev. Mod. Phys. 75, 237 (2003). 